\title{
Passive avoidance in goldfish: Lack of evidence for stimulus specificity
}

\author{
D. J. ZERBOLIO, JR. and L. L. WICKSTRA \\ University of Missouri, St. Louis, Missouri 63121
}

\begin{abstract}
Goldfish, acquiring a passive avoidance response, showed substantially fewer responses in trial intervals than did their yoked controls. A passive procedure, where US reinforcement occurred immediately upon response, produced superior avoidance acquisition to a punishment procedure, where, if response occurred at any time during the trial interval, US reinforcement was administered at the end of the interval. This finding is consistent with the traditional delay of reinforcement gradient. Although goldfish acquired the passive avoidance response, it appeared to be situationally generalized and not stimulus specific, as indicated by a lack of differences between animals trained with a CS and those trained without a CS during trial periods. Also, response suppression between the trial periods was shown by the general ITI response rate suppression. These findings, as well as others showing marked differences between the behavior of goldfish and that of other standard laboratory animals, strongly recommend considerable hesitancy in conclusions of what and how this organism learns.
\end{abstract}

In earlier work, Zerbolio and Wickstra (1978a) found that, in a conditioned avoidance task with a dark intertrial interval (ITI) and a sudden illumination onset conditioned stimulus (CS), goldfish, whether trained classically or instrumentally, showed high and comparable acquisition rates. Further work found that when the ITI was illuminated and a color change was used as the CS, classically trained animals showed no significant increase in performance with training, whereas the instrumentally trained animals continued to do so (Zerbolio \& Wickstra, 1978b). The initial results were consistent with earlier work by Woodard and Bitterman (1973), who concluded that goldfish acquire the conditioned avoidance response (CAR) via a classical process; the second study was consistent with the phototactic explanation offered by Zerbolio and Wickstra (1978b). The study using an illuminated ITI also found that, although pseudoconditioning groups displayed no increase in CAR level with training, other controls (CS only and time lapse) suggested by Jensen (1961) and Kimble (1967) showed very high CAR rates and significant acquisition-comparable to the instrumentally trained subjects. It was concluded that the unpaired pseudoconditioning procedure does not represent everything other controls might do and thus does not seem to be an appropriate learning control for this animal in this apparatus. The finding of the high CS-only and time-lapse control rates also makes any interpretation of learning difficult; it is unclear whether subjects are actually learning an active CAR or, following habituation, simply returning to the unexpectedly high performances shown by the CS-only and time-lapse controls. However, the fact that the controls respond at such high rates could allow the suppression of behavior (namely, the acquisition of a passive avoidance response) to be clearly differentiated from the high control levels
(Zerbolio \& Wickstra, 1978b). Woodard and Bitterman (1973) ran a form of passive avoidance: If the subject responded in the CS interval, an unconditioned stimulus (US) was administered at the end of the interval. This "punishment" procedure imposes a variable delay of reinforcement between subjects' response and reinforcement. Another form of passive avoidance occurs when a subject is reinforced immediately upon response with a zero delay between response and reinforcement. The zero-delay procedure is expected to produce superior acquisition over the variable-delay procedure (Kimble, 1961). Woodard and Bitterman (1973) instituted their punishment procedure after 6 days of other training and did not run CS-termination, CS-nontermination, and no-CS conditions. These are examined in the present $=$ study. Additionally, Zerbolio and Wickstra (1978a, $1978 \mathrm{~b}$ ) found that animals responded more often in the control groups under ITI illumination conditions. The higher response rate militates against acquisition of a passive avoidance response compared to a dark ITI condition. These two conditions were also examined. Yoked controls for all groups were run to determine if the frequency and/or patterning of stimuli alone account for the behavior observed.

\section{METHOD}

\section{Subjects}

The subjects were $2405-6 \mathrm{~cm}$ goldfish obtained from Ozark Fisheries. The fish were fed daily, housed in 30-gal aquaria until $48 \mathrm{~h}$ prior to use, and then housed in individual $7.5 \times 11.5 \mathrm{x}$ $12.5 \mathrm{~cm}$ deep individual aquaria. All housing was well aeriated and filtered, with temperature maintained at $21.1^{\circ} \mathrm{C}$ and $\mathrm{pH}$ held at $7 \pm .1$ at all times.

\section{Apparatus}

The apparatus was the same as that used by Zerbolio and Wickstra (1978a, 1978b). The CS (blue) and ITI (green) illumi- 
nation was provided by $7-\mathrm{W} 110$ ac lamps mounted at both ends of the running tanks and diffused through translucent plastic plates between tank end and bulb. The US, delivered via stainless steel plates affixed to interior side walls, was a $200-\mathrm{msec}$ 7.5-V ac shock (Zerbolio \& Wickstra, 1975). All events were programmed and recorded via appropriate circuitry.

\section{Procedure}

A factorial arrangement of 3 CS conditions, 2 ITI illumination conditions, and 2 training procedures constituted the 12 experimental groups. A yoked control was tested for each of these, making a total of 24 groups with 10 subjects each. Subjects were given 20 trials/day with a VI 60-sec ITI for 5 consecutive days. A trial for the experimental groups was a 10 -sec period that was monitered for shuttling response. If the subject responded during the $10-\mathrm{sec}$ period, the appropriate contingencies (listed below) occurred. The three CS conditions were CS termination contingent on subject's response (CSc), CS termination not contingent (independent of) subject's response (CSnc), and no CS. The ITI illumination conditions were with green illumination during the ITI and a color change to blue for a CS or no ITI illumination and a sudden increase (blue) of illumination for the CS. Training procedures were passive (PAS) or punishment (PUN). With the PAS procedure, subjects that responded during the 10 -sec trial interval were immediately administered a $200-\mathrm{msec} 7.5-\mathrm{V}$ ac US. With the PUN procedure, subjects that responded during the $10-\mathrm{sec}$ trial interval were administered the US at the end of the interval. The PUN procedure imposed a variable delay of reinforcement between response and reinforcement. For both procedures, there was a maximum of one US per trial. Additionally, each experiment subject (master) had its own yoke, which received identical stimulation but exercised no control over that stimulation. The number of $10-\mathrm{sec}$ periods in which at least one response occurred and the ITI response rate were recorded for all subjects.

\section{RESULTS AND DISCUSSION}

A 2 by 2 by 3 by 2 by 5 repeated ANOVA for mean number of $10-\mathrm{sec}$ trial intervals in which a response occurred was calculated. Means are shown in Figure 1. The yoked animals responded in many more 10 -sec trial intervals than did the master experimental animals $\left[\mathrm{F}(1,216)=146.976, \mathrm{p}<.01, \omega^{2}=.2442\right]$. Additionally, there was a difference due to ITI illumination $\left[\mathrm{F}(1,216)=7.894, \mathrm{p}<.01, \omega^{2}=.0114\right]$, and a PAS/ PUN by Master/Yoke interaction was found $[F(4,864)=$ $\left.22.164, p<.01, \omega^{2}=.0225\right]$. All other effects were small, accounting for less than $1 \%$ of the total variance, and are not reported (Vaughan \& Corballis, 1969).

As is obvious from Figure 1, goldfish, by comparison to their yoked controls, do acquire a passive avoidance response. It is interesting to note that, although the yoked subjects received the same number of US presentations as their respective masters, they showed an increase in the number of trial intervals in which a response occurred with "training," whereas the passive animals did not. The yoked subjects' performance curves have the typical negative acceleration characteristic of learning curves and look very similar to the curves found for CS-only and time-lapse control procedures earlier (Zerbolio \& Wickstra, 1978a, 1978b). The finding that all passively trained animals showed a suppression

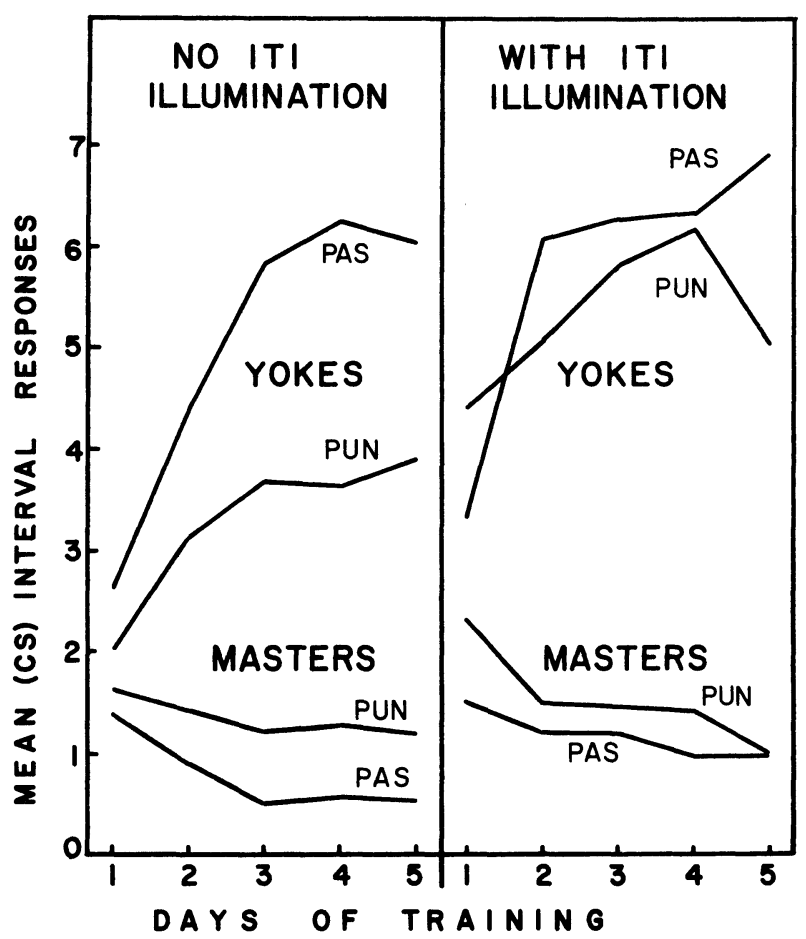

Figure 1. The mean number of CS intervals in which at least one shuttle response occurred, over the 5 days of training. Performance for the experimental master subjects and their yoked controls, for the with- and no-ITI conditions, and for the zero delay of reinforcement or passive (PAS) and variable delay of reinforcement or punishment (PUN) procedures are shown.

of response over training, or at the very least, no increase, clearly indicates that the animals acquired a passive avoidance response. Further, the PAS training procedure was superior to the PUN procedure, indicating that, with a zero delay of reinforcement, goldfish showed acquisition superior to that with the delayed reinforcement procedure. This is consistent with traditional learning expectations (Kimble, 1961, 1967). Obviously, the acquired response suppression is a responsecontingent phenomenon: The yoked subjects, although receiving an equal number of shocks, showed performance substantially above the experimental master animals. The disturbing finding about these data is that there was no effect due to the CS manipulation. Specifically, groups with a CS did not differ among themselves and, more importantly, did not differ from comparable groups with no CS at all insofar as their passive avoidance performance. Obviously, the passive or punishment response contingency is sufficient to generally depress response rate, independent of the CS condition. This is supported by a comparable five-way ANOVA of the square-root-transformed ITI shuttle rate data showing only a main effect for master/yoke differences $\left[\mathrm{F}(1,216)=106.12, \mathrm{p}<.01, \omega^{2}=.2144\right]$ and a Master $/$ Yoke by Days of Training interaction $[\mathrm{F}(4,864)=26.35$, $\left.\mathrm{p}<.01, \omega^{2}=.027\right]$, indicating that the yoked subjects 
increased their ITI shuttle rate over days of training, whereas the experimental masters did not.

At the very best, we can conclude that goldfish are sensitive to the passive avoidance contingency with respect to the US presentation, but we found no evidence indicating that this acquisition is CS stimulus specific. Rather, it appears to be a generalized response suppression phenomenon. It is tempting to conclude that, since the response suppression shown here by the passively trained animals is a response-contingent phenomenon, these animals learned by an instrumental process. But the finding of high response rates shown by control conditions in the present study and in earlier work (Zerbolio \& Wickstra, 1978a, 1978b) suggests that conclusions about the process or processes by which goldfish acquire either active or passive avoidance responses, especially in one-stimulus paradigms, may be both preliminary and unwise. Obviously further work is indicated.

\section{REFERENCES}

Jensen, D. D. Operationism and the question "Is this behavior learned or innate?" Behavior, 1961, 17, 1-8.
Kimble, G. A. Hilgard and Marquis' conditioning and learning (2nd ed.). New York: Appleton-Century-Crofts, 1961.

Kimble, G. A. Foundations of conditioning and learning. New York: Appleton-Century-Crofts, 1967.

Vaughan, B. M., \& Corballis, M. C. Beyond tests of significance: Estimating strength of effects in selected ANOVA designs. Psychological Bulletin, 1969, 72, 204-213.

Woodard, W. R., \& BitTerman, M. E. Pavlovian analysis of avoidance conditioning in the goldfish (Carassius auratus). Journal of Comparative and Physiological Psychology, 1973, 82, 123-129.

Zerbolio, D. J., \& Wickstra, L. L. The effect of power (US intensity $x$ US duration) on shuttlebox avoidance acquisition in goldfish. Bulletin of the Psychonomic Society, 1975, 5, 345-347.

Zerbolio, D. J., \& Wickstra, L. L. Goldfish avoidance acquisition: Is the process classical, instrumental, or a phototaxis? Bulletin of the Psychonomic Society, 1978, 11, 321-323. (a)

Zerbolio, D. J., \& Wickstra, L. L. Does elimination of a negative phototaxis eliminate CAR acquisition in goldfish? Bulletin of the Psychonomic Society, 1978, 11, 324-326. (b)

(Received for publication March 1, 1978.) 